\title{
Impact of Slow Yogic-Type of Breathing on Physiological Effects of Breath Holding Time
}

\author{
Rajajeya Kumar $\mathbf{M}^{1^{*}}$ and Madanmohan $\mathrm{T}^{2}$ \\ ${ }^{1}$ Department of Physiology, Chennai, Medical College Hospital and Research Centre, Irungalur, Trichy, Tamilnadu, India \\ ${ }^{2}$ Department of Physiology, Mahatma Gandhi Medical College \& Research Institute, Pondicherry -607 402, India
}

"Corresponding author: Rajajeya Kumar M, Assistant Professor, Department of Physiology, Chennai Medical College Hospital and Research Centre, Irungalur, Trichy, Tamilnadu, India, Tel: 09751382650; E-mail: rajakumar60@gmail.com

Received date: Mar 12, 2015 Accepted date: Mar 13, 2015 Published date: Mar 16, 2015

Copyright: ( 2015 Rajajeya Kumar M, et al. This is an open-access article distributed under the terms of the Creative Commons Attribution License, which permits unrestricted use, distribution, and reproduction in any medium, provided the original author and source are credited.

\section{Introduction}

The modern concept of Yoga is explained in terms of integration, harmony and balance. This can be the effective methods in alternative and/or complementary medicine for the management of chronic functional disorders. It contributes to the holistic health through promotive, preventive and curative method [1].

\section{Homoeostatic effect}

Different yogic techniques bring control effect, which means the minor changes of the internal environment of the system towards a state of normal and optimal balance. This homoeostasis is maintained by the balance of sympathetic and Para-sympathetic component of autonomic nervous system as well as by the endocrinal system.

\section{How to practice breath holding techniques?}

Breath holding techniques will be introduced slowly and with proper guidance and experience. The patients should advise to practice the technique in the absence known cardiac problem. These techniques appear to pose no threat when done properly and introduced gradually for normal subjects.

\section{Cardiovascular effects of breath holding}

The heart rate decreases if the breath is held after maximum inhalation and increases after maximum exhalation due to pressure against the closed airway. Strong exhalation pressure, positive intrapulmonary pressure can increase heart rate above the baseline [2]. The reduced peripheral blood flow is accentuated by exhalation pressure during breath holding.

\section{Breathe holding and Sympatho-Vagal balance}

Breath holding and the dive response can initiate both parasympathetic and sympathetic cardiovascular responses. Heart rate slowing is apparently mediated by the parasympathetic system whereas the reduced peripheral blood flow results from increased peripheral sympathetic activity. Finally, breath holding helps to restore the Sympatho-Vagal Balance is analyzed by a simple, noninvasive method known as Herat rate variability (HRV) [3].

\section{Impact of psychological factor on breath holding}

Psychological factors can override or enhance the autonomic responses to breathe holding [4]. Mental distraction or preoccupation during breath holding or the dive response can increase and decrease the cardiovascular responses to breathe holding.

\section{Precautions for practicing breath holding in cardiac patients}

Breath holding and the dive response can induce a variety of cardiac arrhythmias in patients with significant pre-existing heart abnormalities. The strong parasympathetic cardiac stimulation from the dive response has been successfully and safely used as a means of halting paroxysmal atrial tachycardia, but requires extreme caution in patients susceptible to ventricular abnormalities because dangerous arrhythmias can be induced [5].

\section{Conclusion}

Yoga can be a practical tool to Body-Mind integration for the holistic healing and positive health. A systematic attempt to implement Yoga into formal healthcare system may bring great benefits to general public. For this, healthcare professionals may have general knowledge of Yoga and the right perspective of its application.

\section{Further Research}

Effect of advanced breath holding practices on various physiological parameters can be investigated for future research study.

\section{References}

1. Digambar, Swami, Kokaje, Raghunathshastri (1984) Lesson V: Yoga Mimansa 23: 1.

2. Paulev P, Honda Y, Sakakibara Y, Morikawa T, Tanaka Y, et al. (1988) Brady- and tachycardia in light of the valsalva and mueller maneuver. Japanese Journal of Physiology 38: 507-517.

3. Rajajeyakumar M, Prakash ES, Pawar GS, Narkunaraja, Tamizharasan T, et al. (2014) Is Any Significant Impact of Average RR Interval on Prognostic Ability of Sympatho Vagal Homeostasis. J Bioengineer \& Biomedical Sci 4: 121.

4. Elsner R, Gooden B (1983) Diving and asphyxia. Cambridge: Cambridge University Press.

5. Whayne TF, Killip T (1967) Simulated diving in man: Comparison of facial stimuli and response in arrhythmia. Journal of Applied Physiology 22: $800-807$. 